\title{
Supporting Retention in HIV Care: Comparing In-Person and Telehealth Visits in a Chicago-Based Infectious Disease Clinic
}

\author{
Arianna I. Boshara ${ }^{1}$ (D) Megan E. Patton ${ }^{2} \cdot$ Bijou R. Hunt $^{1} \cdot$ Nancy Glick $^{1} \cdot$ Amy K. Johnson $^{3}$ \\ Accepted: 21 January 2022 / Published online: 3 February 2022 \\ (c) The Author(s), under exclusive licence to Springer Science+Business Media, LLC, part of Springer Nature 2022
}

\begin{abstract}
The COVID-19 pandemic has created increased need for telehealth appointments. To assess differences in appointment adherence for telehealth compared to in-person HIV medical care visits, we conducted a cross-sectional study of patients receiving HIV care in a safety-net hospital-based outpatient infectious disease clinic in a large urban area (Chicago, IL). The sample $(\mathrm{N}=347)$ was predominantly Black $(\mathrm{n}=251)$ and male $(62.5 \%, \mathrm{n}=217)$; with a mean age of 44.2 years. Appointment attendance was higher for telehealth (78.9\%) compared to in-person (61.9\%) appointments. Compared to patients without drug use, those with drug use had 19.4 percentage point lower in-person appointment attendance. Compared to those with stable housing, those in unstable housing arrangements had 15.0 percentage point lower in-person appointment attendance. Telehealth as a modality will likely have some staying power as it offers patients newfound flexibility, but barriers to telehealth need to be assessed and addressed.
\end{abstract}

Keywords Telemedicine $\cdot$ Telehealth $\cdot$ HIV $\cdot$ Adherence

\section{Introduction}

The COVID-19 pandemic has posed significant challenges to healthcare delivery, necessitating the rapid adoption of telehealth services (the delivery of healthcare services using technologies for information and communication) during phases which required restricting in-person visits. [1] Prior to the pandemic, telehealth was narrowly implemented to increase access for patient populations with reduced access to care [2, 3], including to HIV care. [4] While many providers across the country did offer telehealth services during the pandemic, wide scale implementation and adoption of telehealth for HIV medical care has not occurred. Further, studies on telehealth for HIV medical care in the context of the COVID-19 pandemic are limited. [5-7] Prior studies suggest

Arianna I. Boshara

arianna.boshara@sinai.org

1 Sinai Infectious Disease Center, Sinai Chicago, $1500 \mathrm{~S}$. Fairfield Ave., Chicago, IL, USA

2 Lewis Katz School of Medicine at, Temple University, 3500 N. Broad St., Philadelphia, PA, USA

3 Feinberg School of Medicine \& Ann \& Robert H. Lurie Children's Hospital, Northwestern University, 225 East Chicago Avenue, Chicago, IL, USA that by mitigating barriers associated with in-person visits, remote delivery of HIV services may improve appointment adherence, impact viral suppression, and ultimately support health outcomes among people living with HIV and prevent onward transmission. [8-11] A recent study of 371 people living with HIV found that $57 \%$ of participants were more likely to use telehealth for their HIV visits compared to inperson visits, demonstrating both feasibility and acceptability. [8], 12].

Retention in HIV medical care is critical to maintaining health and achieving HIV viral suppression. [13] However, the CDC estimates that only 50\% of persons living with HIV are retained in care and 57\% have a suppressed viral load. [14] People living with HIV who are engaged in regular HIV care demonstrate better ART adherence, are more likely to achieve viral suppression, and are more likely to sustain positive health outcomes. [15-17] Factors such as housing, substance use, transportation, and competing priorities have been identified as barriers for people living with HIV to be retained in medical care. [18] Additionally, patients who are Black and/or have low SES are at higher risk of missing their appointments and falling out of care due to systemic barriers and the impact of racism. [19] Telehealth services may be a strategy to reduce barriers to retention and increase access to HIV medical care. [20] However, important limitations 
of telehealth also must be considered, including potential difficulty obtaining HIV and other STI screening [8], and accessibility of telehealth given connectivity and device requirements. [21, 22].

As was the case across the United States, Sinai Chicago, an urban, safety-net health system, was heavily affected during the peak of the COVID-19 pandemic. In March 2020, the Mount Sinai Hospital Infectious Disease Clinic (MSHIDC) rapidly transitioned to a virtual healthcare delivery model, during which time all appointments were converted to telehealth visits. In this retrospective study, we investigate whether there is a difference in adherence to in-person vs. telehealth appointments among patients living with HIV during the COVID-19 pandemic at an outpatient, hospitalbased infectious disease (ID) clinic in a large urban area.

\section{Materials and Methods}

\section{Setting}

Sinai Chicago, Illinois' largest private safety-net health system, serves the west and southwest sides of Chicago. These under-resourced communities are made up of predominantly racial and ethnic minority populations [23], which suffer a disproportionate burden of HIV. [24] The majority of persons in the Sinai Chicago service area, as well as patients seen at Sinai Chicago, are un- or under-insured, non-Hispanic Black or Hispanic, and many are monolingual Spanish-speaking. [25].

Sinai Chicago includes 3 hospitals and 14 outpatient clinics across the system's service area. MSHIDC is an outpatient hospital-based clinic, providing comprehensive ID care specializing in HIV care services.

\section{Study Population and Source of Data}

We conducted a cross-sectional study of all MSHIDC patients 18 years of age and older who had (a) at least one HIV telehealth visit scheduled between March 2020 and May 2021 and/or (b) at least one HIV in-person visit scheduled between January 2017 and May 2021. De-identified appointment data was downloaded from Meditech, the electronic medical record (EMR). The Mount Sinai Hospital Institutional Review Board approved this project (protocol \#16-14).

\section{Explanatory Variables}

Explanatory variables included patient demographics (sex, age, race/ethnicity) and HIV-related risk factors (substance use-drug and alcohol; men who have sex with men (MSM), and unstable housing). Reference groups were: sex: female; age: 18-24; race/ethnicity: non-Hispanic White. Age was treated as a continuous variable in regression models. MSM was based on either self-report by individuals who identify as MSM or cis-gender men reporting homosexual sex. Substance use-drug captured patients who reported past or current drug use. Substance use-alcohol captured patients who reported past or current alcohol use. Unstable housing captured patients who reported current unstable housing arrangements.

\section{Outcome Variables}

The primary outcome variables in this analysis were telehealth appointment attendance (\%) and in-person appointment attendance $(\%)$. Telehealth appointment attendance was calculated by dividing the number of telehealth appointments attended by the number of telehealth appointments scheduled. In-person appointment attendance was calculated by dividing the number of in-person appointments attended by the number of in-person appointments scheduled. All appointments included in the analysis were for visits with an ID physician for routine HIV care (no urgent care or unscheduled visits). Telehealth visits were ideally conducted using doxy.me, a free and secure platform that allowed the patient to connect via a link sent via text message, and which required no download on the patient's end; however, providers could conduct the visit over the phone when any barriers prevented connecting via the platform.

\section{Analysis}

In order to understand differences in overall appointment attendance between in-person and telehealth (unpaired data), we calculated the average attendance across the sample for each appointment type and used a t-test (test statistic: $t$ ) to detect differences between in-person and telehealth attendance. This same approach was applied to examine differences in appointment attendance by demographic and risk groups. To detect differences across demographic and risk groups within appointment type (unpaired data), we calculated the average attendance across the sample for each appointment type and used a Mann-Whitney test (test statistic: $U$ ) to indicate statistically significant differences between groups for each appointment type. We used $p<0.05$ to indicate statistical significance across all analyses.

To detect differences in appointment attendance between in-person and telehealth for patients who had both appointment types (paired data), we calculated attendance at the individual record level and compared in-person to telehealth attendance using the Wilcoxon test (test statistic: $W$ ) to indicate statistically significant differences in attendance. This same approach was applied to examine differences in 
appointment attendance by demographic and risk groups (test statistic: $t$ ).

Finally, we ran linear regression models in order to understand the predictors of appointment attendance (\%) by visit type (test statistic: $t$ ). The first model for each visit type included a block of demographic variables; the second model for each visit type included both demographic and risk factor variables.

\section{Results}

The sample population $(\mathrm{N}=347)$ was: $72.3 \%$ Black $(n=251), 19.9 \%$ Hispanic $(n=69), 5.8 \%$ White $(n=20)$, and $2 \%$ Asian $(\mathrm{n}=7)$; predominantly male $(62.5 \%, \mathrm{n}=217)$; with a mean age of 44.2 years (Table 1 ). Forty percent of reporting individuals $(n=229)$ identified as MSM $(n=92 / 229)$, $5 \%$ reported injection drug use (IDU) $(n=16 / 339), 14 \%$ had substance use issues with drugs $(n=48 / 347), 9 \%$ had substance use issues with alcohol $(n=32 / 347)$, and $14 \%$ were in unstable housing situations $(n=36 / 251)$.

Ninety-six percent of the sample had at least one inperson appointment scheduled during the study period $(\mathrm{n}=332)$, while $71 \%$ had at least one telehealth appointment scheduled during the study period $(\mathrm{n}=246)$.

Overall appointment attendance for the full sample (unpaired data) was statistically significantly higher for telehealth appointments $(78.9 \%)$ than for in-person appointments $(61.9 \%)(t=-5.83, \mathrm{p}<0.001)$. Statistically significantly higher telehealth appointment attendance compared to in-person attendance was observed among Black $(78.2 \%$ vs. $58.3 \%, t=-5.59, \mathrm{p}<0.001)$; female $(82.7 \%$ vs. $63.1 \%$, $t=-4.43, \mathrm{p}<0.001)$; male $(76.3 \%$ vs. $61.1 \%, t=-3.94$, $\mathrm{p}<0.001)$; and $25-34(72.5 \%$ vs. 59.7\%, $t=-2.34$, $\mathrm{p}=0.021), 35-44(80.2 \%$ vs. $60.7 \%, t=-3.14, \mathrm{p}=0.002)$, $45-54(82.4 \%$ vs. $64.4 \%, t=-2.88, \mathrm{p}=0.005), 65+(89.5 \%$ vs. $60.9 \%, t=-2.90, \mathrm{p}=0.006)$ year old patients. Across risk categories, attendance was higher for telehealth than inperson appointments, regardless of MSM, substance use, or housing status. IDU was the only risk category for which the higher telehealth appointment attendance (66.7\%) was not statistically significantly higher than the in-person appointment attendance (40.1\%); this was likely due to small sample size for IDU $(n=16)$. The majority of differences observed in the full sample (unpaired data) held for the analysis of paired data as well.

Examining differences across groups within in-person appointments (unpaired data), we observe statistically significantly lower in-person appointment attendance for Black patients $(58.3 \%)$ compared to all other patients $(72.0 \%)$ ( $U=-3.34, \mathrm{p}=0.001)$; patients with IDU (40.1\%) compared to non-IDU $(62.5 \%)(U=2.13, \mathrm{p}=0.033)$; patients with drug use $(38.2 \%)$ compared to those without $(65.8 \%)$
$(U=4.89, \mathrm{p}<0.001)$; patients with alcohol use $(50.6 \%)$ compared to those without $(63.0 \%)(U=2.13, \mathrm{p}=0.033)$; patients with unstable housing arrangements $(48.0 \%)$ compared to those with stable housing (70.8\%) $(U=4.21$, $\mathrm{p}<0.001)$. In the paired data, the patterns of lower in-person compared to telehealth attendance were only observed among patients with drug use and unstable housing. No statistically significant cross-group differences in telehealth appointment attendance were observed in either the paired or unpaired data.

In regression model 1 for in-person appointment attendance, Hispanic ethnicity was the only demographic variable found to be statistically significant (beta $=19.1, t=2.3$, $\mathrm{p}=0.020$ ) (Table 2). With the addition of risk factor variables in model 2, Hispanic ethnicity is no longer significant. Substance use - drug and unstable housing emerge as statistically significant predictors of in-person appointment attendance with both variables demonstrating a negative relationship with attendance. Compared to patients without drug use, those with drug use have 19.4 percentage point lower in-person appointment attendance $(t=-3.5$, $\mathrm{p}=0.001)$. Compared to those with stable housing, those in unstable housing arrangements have 15.0 percentage point lower in-person appointment attendance $(t=-3.0$, $\mathrm{p}=0.003$ ). None of the demographic or risk factor variables were significantly associated with telehealth appointment attendance.

\section{Discussion}

One of the four strategies to end the HIV Epidemic is to rapidly and effectively treat people with HIV to reach sustained viral suppression. [20] Retention in HIV care is critical to sustaining viral suppression. Addressing methods to increase the number of people who are virally suppressed is a necessity to end the epidemic. Telehealth provides patients flexibility and autonomy, expanding options for those receiving ongoing HIV care. The COVID-19 pandemic has prompted a shift to telehealth options as a means to continue healthcare delivery despite the need for physical distancing. [1] The results of our study indicate that overall HIV medical appointment attendance was higher with telehealth appointments compared to in-person appointments, indicating that telehealth should continue to be offered as an option to increase retention in HIV care beyond a pandemic setting.

Barriers to remaining in HIV medical care exist. Our results indicate that those who endorsed drug use or unstable housing had a lower rate of attending in-person appointments compared to those who did not endorse drug use or had stable housing, yet these same relationships were not seen when we examined telehealth appointment adherence. Telehealth visits may be a method to overcome barriers 


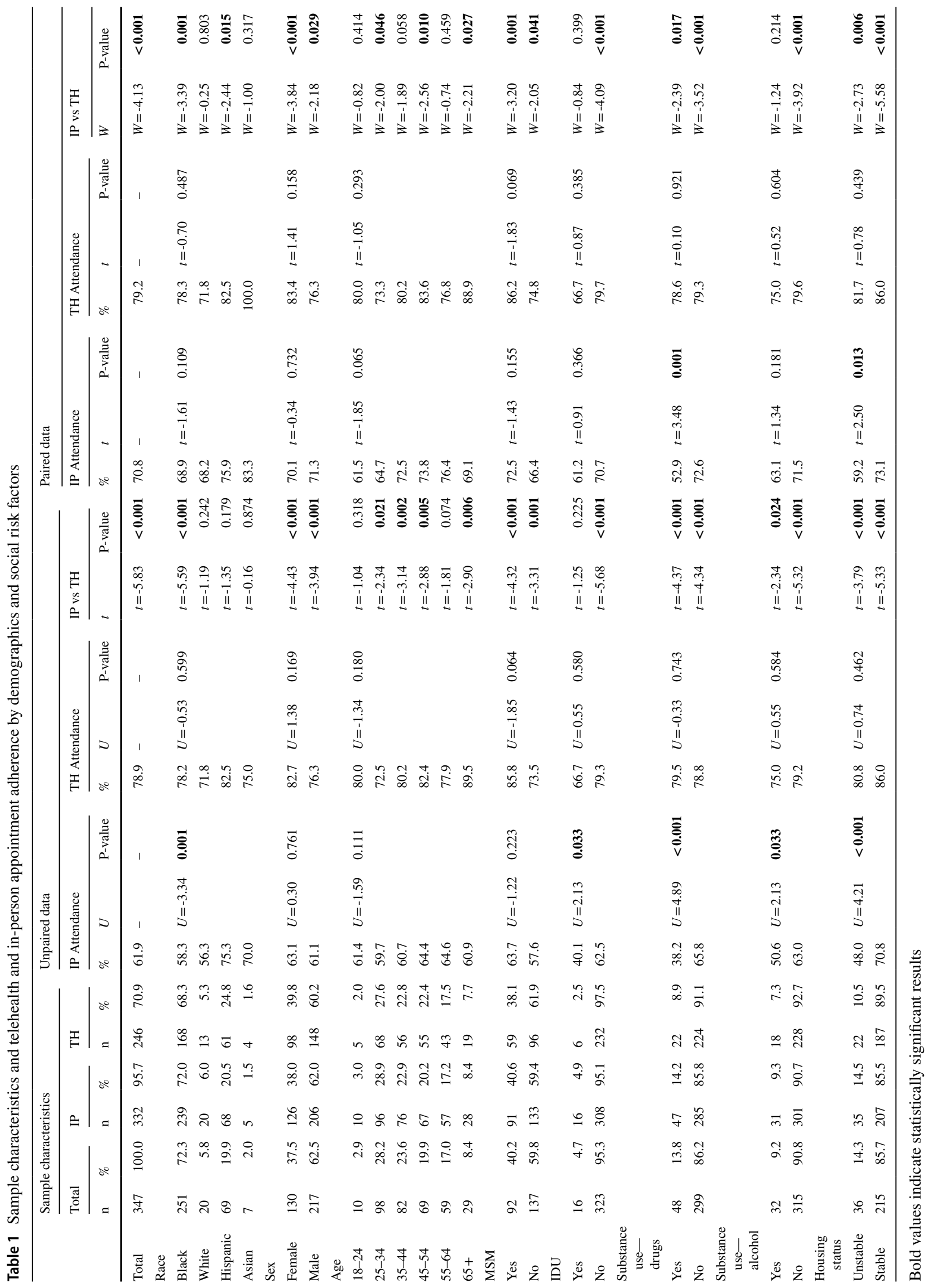


Table 2 Predictors of telehealth and in-person appointment adherence

\begin{tabular}{|c|c|c|c|c|c|c|c|c|c|c|c|c|}
\hline & \multicolumn{6}{|c|}{ IP attendence } & \multicolumn{6}{|c|}{$\mathrm{TH}$ attendence } \\
\hline & \multicolumn{3}{|c|}{ Model 1} & \multicolumn{3}{|c|}{ Model 2} & \multicolumn{3}{|c|}{ Model 1} & \multicolumn{3}{|c|}{ Model 2} \\
\hline & $\beta$ & $\mathrm{t}$ & $\mathrm{P}$-value & $\beta$ & $\mathrm{t}$ & P-value & $\beta$ & $\mathrm{t}$ & P-value & $\beta$ & $\mathrm{t}$ & P-value \\
\hline \multicolumn{13}{|l|}{ Demographics } \\
\hline Female & Ref & - & - & Ref & - & - & Ref & - & - & Ref & - & - \\
\hline Male & -3.1 & -0.8 & 0.400 & 5.4 & 1.6 & 0.114 & -6.1 & -1.3 & 0.213 & -2.4 & 0.6 & 0.585 \\
\hline White & Ref & - & - & Ref & - & - & Ref & - & - & Ref & - & - \\
\hline Black & 1.9 & 0.3 & 0.803 & -4.4 & -0.6 & 0.540 & 5.6 & 0.5 & 0.604 & 5.8 & 0.6 & 0.572 \\
\hline Hispanic & 19.1 & 2.3 & 0.020 & 5.0 & 0.6 & 0.522 & 10.7 & .9 & 0.352 & 9.1 & 0.9 & 0.398 \\
\hline Asian & 14.5 & 0.9 & 0.367 & -3.3 & -0.1 & 0.901 & 2.9 & 0.1 & 0.891 & 14.9 & 0.6 & 0.539 \\
\hline Age & 0.1 & 0.4 & 0.668 & 0.2 & 1.9 & 0.061 & 0.2 & 1.4 & 0.159 & 0.8 & 0.5 & 0.65 \\
\hline \multicolumn{13}{|l|}{ Risk factors } \\
\hline MSM & - & & - & 0.0 & 0.5 & 0.599 & - & & - & 0.1 & 1.6 & 0.105 \\
\hline Sub use: drug & - & & - & -19 & -3.5 & 0.001 & - & & - & 1.2 & 0.2 & 0.878 \\
\hline Sub use: alcohol & - & & - & -8.6 & -1.5 & 0.130 & - & & - & -10.1 & -1.3 & 0.212 \\
\hline Unstable housing & - & & - & -15.0 & -3.0 & 0.003 & - & & - & -3.7 & -0.5 & 0.613 \\
\hline Mode P & 0.06 & & & 0.000 & & & 0.394 & & & 0.505 & & \\
\hline
\end{tabular}

patients face, such as the impact of drug use and unstable housing, to retention in HIV medical care.

Important concerns related to telehealth must be noted. As broadband internet has been developed, disparities in access have become a prevalent public health issue, where underserved communities more often lack access. [21] Participation in telehealth services might prove difficult if patients cannot access a stable high-speed internet connection for video feeds. Further, access to a mobile phone or computer is needed, as is the ability to download healthcare applications or access webapps for telehealth visits. Technological barriers may be more pronounced in those who are older or in rural settings, as a result of the digital divide. [22] Despite this potential and the fact that in 2020, less than $7 \%$ of people living with HIV over age 50 reported engaging in telehealth, in $202180 \%$ of the same demographic engaged in telehealth services during the pandemic.[22] Flexibility in telehealth services may also be warranted; Dandachi et al. [8] report the use of audio only and phone calls for HIV care telehealth visits when patients were unable to access video feed via Zoom teleconference. [8] Thus, patients that might benefit most from telehealth HIV medical care are also those who may face barriers to accessing services and need additional support. [21] Future research should explore the uptake of telehealth visits among those populations who may experience the most internet, software, and device barriers and investigate needed support for uptake. One possibility would be to explore reimbursement for phone-based telehealth visits as a solution to the technology challenges, but also if current telehealth laws enacted during the pandemic expire.
As COVID-19 mitigation mandates loosen with vaccine uptake, the continued availability of telehealth appointments for HIV care is in question. Prospective evaluation is needed to determine the impact of appointment adherence, by type of appointment, in an environment in which both in-person and telehealth appointments are being offered.

\section{Limitations}

This study should be interpreted with several limitations in mind. First, it was conducted in a safety-net hospital in a large urban area with a sample population that was predominantly Black and male. Results may not generalize to other populations or settings. However, the distribution of the HIV epidemic in the US is disproportionately male and Black, thus the study population reflects this. Second, the study was conducted during the COVID-19 pandemic. Adherence to telehealth versus in-person appointments can be driven by public health recommendations specific to lockdown mitigation measures. Additionally, telehealth visits became an insurance-billable service during the pandemic, increasing wide scale availability. Third, telehealth visits occurred via a variety of modalities and our ability to be flexible in how we reached the patient may have impacted our results for telehealth adherence. Fourth, we used an explanatory variable that indicates past or current drug or alcohol use. These indicators may present issues with temporality as we are unable to differentiate between past and current use. Finally, data was collected from 
the electronic medical record system, which can contain missing values; however, chart reviews were performed to minimize missing data.

\section{Conclusion}

The COVID-19 pandemic has introduced an unexpected opportunity to expand the ways in which HIV care is offered in the United States. Telehealth as a modality will likely have some staying power as it offers patients newfound flexibility and autonomy. Barriers to accessing telehealth will need to be addressed to ensure equity in uptake and benefit.

Author Contributions $\mathrm{AB}$ conducted the analysis for this study and led the writing of the Abstract, Discussion and Conclusion sections, and contributed to the writing of the Introduction, Methods, and Results sections. MP performed background research that guided the development of the research questions and conducted extensive data collection and cleaning processes. BH conceived of this cross-sectional study. She helped to conceptualize research questions. She led the writing of the Methods section and the Results section, and contributed to the writing of the Introduction, Discussion, and Conclusion sections. She provided overall guidance on analysis and review. NG guided the development of the study and provided overall review. AJ guided and oversaw the analytical methods of this study and led the writing of the Introduction Section. She contributed to the writing of the Methods, Results, Discussion, and Conclusion sections.

Funding No funds, grants, or other support was received.

Availability of data and material All data and materials as well as software application or custom code support published claims and comply with field standards.Code availability All code and analysis was written and performed in Stata (version 14.1).

\section{Declarations}

Conflict of interest The authors have no conflicts of interest to declare that are relevant to the content of this article.

Consent to participate The IRB approved the request for waiver or alteration of HIPAA authorization for research and subsequent consent to participate.

Consent for publication The IRB approved the request for waiver or alteration of HIPAA authorization for research and subsequent consent to publish.

Ethical approval The Mount Sinai Hospital Institutional Review Board approved this project (protocol \#16-14).

\section{References}

1. Kruse CS, Krowski N, Rodriguez B, Tran L, Vela J, Brooks M. Telehealth and patient satisfaction: a systematic review and narrative analysis. BMJ Open. 2017;7(8):1016242.

2. Xu T, Pujara S, Sutton S, Rhee M. Telemedicine in the management of type 1 diabetes. Prev Chronic Dis. 2018;15:E13.

3. Wood CL, Clements SA, McFann K, Slover R, Thomas JF, Wadwa RP. Use of telemedicine to improve adherence to American diabetes association standards in pediatric type 1 diabetes. Diabetes Technol Ther. 2016;18(1):7-14.

4. Ohl ME, Richardson K, Rodriguez-Barradas MC, Bedimo R, Marconi V, Morano JP, et al. Impact of availability of telehealth programs on documented HIV viral suppression: a cluster-randomized program evaluation in the veterans health administration. Open Forum Infect Dis. 2019;6(6):ofz206.

5. Budak JZ, Scott JD, Dhanireddy S, Wood BR. The impact of COVID-19 on HIV care provided via telemedicine-past, present, and future. Curr HIV/AIDS Rep. 2021;18(2):98-104.

6. Yelverton V, Qiao S, Weissman S, Olatosi B, Li X. Telehealth for HIV care services in south carolina: utilization, barriers, and promotion strategies during the COVID-19 pandemic. AIDS Behav. 2021;25:3909-21

7. Auchus IC, Jaradeh K, Tang A, Marzan J, Boslett B. Transitioning to telehealth during the COVID-19 pandemic: patient perspectives and attendance at an HIV clinic in San Francisco. AIDS Patient Care STDS. 2021;35(7):249-54.

8. Dandachi D, Freytag J, Giordano TP, Dang BN. It is time to include telehealth in our measure of patient retention in HIV care. AIDS Behav. 2020;24(9):2463-5.

9. Saifu HN, Asch SM, Goetz MB, Smith JP, Graber CJ, Schaberg D, et al. Evaluation of human immunodeficiency virus and hepatitis $\mathrm{C}$ telemedicine clinics. Am J Manag Care. 2012;18(4):207-12.

10. Lee I, Kovarik C, Tejasvi T, Pizarro M, Lipoff JB. Telehealth: Helping your patients and practice survive and thrive during the COVID-19 crisis with rapid quality implementation. J Am Acad Dermatol. 2020;82(5):1213-4.

11. Bakken S. Telehealth: simply a pandemic response or here to stay? J Am Med Inform Assoc. 2020;27(7):989-90.

12. Dandachi D, Dang BN, Lucari B, Teti M, Giordano TP. Exploring the attitude of patients with HIV about using telehealth for HIV care. AIDS Patient Care STDS. 2020;34(4):166-72.

13. Kay ES, Batey DS, Mugavero MJ. The HIV treatment cascade and care continuum: updates, goals, and recommendations for the future. AIDS Res Ther. 2016;13:35.

14. Centers for Disease Control and Prevention. Monitoring selected national HIV prevention and care objectives by using HIV surveillance data-United States and 6 dependent areas, 2019. HIV Surveillance Supplemental Report 2021;26(No.2). http://www.cdc.gov/hiv/library/reports/hiv-surveillance.html. Published May 2021. Accessed [8/9/2021].

15. Tripathi A, Youmans E, Gibson JJ, Duffus WA. The impact of retention in early HIV medical care on viro-immunological parameters and survival: a statewide study. AIDS Res Hum Retroviruses. 2011;27(7):751-8.

16. Yehia BR, French B, Fleishman JA, Metlay JP, Berry SA, Korthuis $\mathrm{PT}$, et al. Retention in care is more strongly associated with viral suppression in HIV-infected patients with lower versus higher CD4 counts. J Acquir Immune Defic Syndr. 2014;65(3):333-9.

17. Shumba $\mathrm{C}$, Atuhaire L, Imakit R, Atukunda R, Memiah P. Missed doses and missed appointments: adherence to ART among adult patients in Uganda. Isrn Aids. 2013;2013:270914.

18. Holtzman CW, Shea JA, Glanz K, Jacobs LM, Gross R, Hines $\mathrm{J}$, et al. Mapping patient-identified barriers and facilitators to 
retention in HIV care and antiretroviral therapy adherence to Andersen's behavioral model. AIDS Care. 2015;27(7):817-28.

19. Israelski D, Gore-Felton C, Power R, Wood MJ, Koopman C. Sociodemographic characteristics associated with medical appointment adherence among HIV-seropositive patients seeking treatment in a county outpatient facility. Prev Med. 2001;33(5):470-5.

20. Key Strategies in the Plan. Office of Infectious Disease and HIV/ AIDS Policy, HHS. Updated May 8, 2020. Accessed August 1, 2021. https://www.hiv.gov/federal-response/ending-the-hiv-epide mic/key-strategies.

21. Bauerly BC, McCord RF, Hulkower R, Pepin D. Broadband access as a public health issue: the role of law in expanding broadband access and connecting underserved communities for better health outcomes. J Law Med Ethics. 2019;47(2):39-42.

22. State of Aging with HIV. Health HIV. Updated July 1, 2021. Accessed August 1, 2021. https://healthhiv.org/wp-content/uploa ds/2021/07/HealthHIV_Second_Annual_State_of_Aging_with_ HIV.pdf.

23. Roesch P, Castro M, Monnard K, et al. Mount Sinai Hospital 2019 Community Health Needs Assessment. Sinai Health System.
Published 2019. Accessed July 20, 2021. https://storage.googl eapis.com/sinai-staging/uplaods/2020/11/8d68fdc8-mount-sinaihospital-2019-chna-.pdf

24. Chicago Department of Public Health. 2019 HIV/STI Surveillance Report. City of Chicago. Published December 2019. Accessed July 29, 2021. https://www.chicago.gov/content/dam/city/depts/ cdph/statistics_and_reports/2019_HIV_STI_REPORT_Final.pdf

25. Hirschtick J, Benjamins M, Homan S. Community Health Counts: Sinai Community Health Survey 2.0. Sinai Urban Health Institute, Sinai Health System. Chicago, IL. March 2017. Accessed July 10, 2021. https://e1f1cf29-5109-4b10-8346-eee7ed0dfa10.files usr.com/ugd/6b5ac9_bee17298461e4e00862737df7b304ca4.pdf.

Publisher's Note Springer Nature remains neutral with regard to jurisdictional claims in published maps and institutional affiliations. 